\title{
Association of overexpression of efflux pump genes with antibiotic resistance in Pseudomonas aeruginosa strains clinically isolated from urinary tract infection patients
}

\author{
Katsumi Shigemura ${ }^{1,2}$, Kayo Osawa ${ }^{2,3}$, Ayaka Kato ${ }^{3}$, Issei Tokimatsu ${ }^{2}$, Soichi Arakawa ${ }^{1,2}$, Toshiro Shirakawa ${ }^{1,3,4}$ \\ and Masato Fujisawa ${ }^{1}$
}

There are several mechanisms for antibiotic-resistant Pseudomonas aeruginosa. The purpose of this study is to investigate the association between the expression of efflux pump-coding genes and antibiotic resistance in $P$. aeruginosa causing urinary tract infections (UTIs). We extracted the RNA from 105 clinical strains of $P$. aeruginosa isolated from UTI patients with full data on antibiotic MICs and assayed real-time quantitative reverse-transcription PCR. We investigated the gene expressions of four resistance nodulation cell division-type multi-drug efflux pump systems (MexAB-OprM, MexCD-OprJ, MexEF-OprN and MexXY $(-O p r A))$ and the correlation of the MICs of nine antibiotics, risk factors and antibiotic resistance-related genes with expressions of mexB, mexC, mexE and mexY. Multivariate statistical data demonstrated a significant relationship between increased expression of mexB or mexC and complicated UTI (Odds ratio $=8.03, P<0.001$ and Odds ratio $=8.86, P=0.032$, respectively). We also found a significant association between the increased expression of $\operatorname{mex} C$ and resistance to levofloxacin (LVFX) (Odds ratio $=4.48, P=0.035$ ). In conclusion, increased expression of $m e x C$ leads to LVFX resistance in $P$. aeruginosa causing UTI. These results contribute to our knowledge of the efflux pump system and antibiotic resistance.

The Journal of Antibiotics (2015) 68, 568-572; doi:10.1038/ja.2015.34; published online 8 April 2015

\section{INTRODUCTION}

Antibiotic resistance in Pseudomonas aeruginosa, one of the most common pathogens in complicated urinary tract infections (UTIs), has spread in the Asia-Pacific region. ${ }^{1-3}$ The overexpression of efflux pumps was reported to contribute to multi-drug resistance in P. aeruginosa. ${ }^{4}$ Most Gram-negative bacteria have the genes for efflux pumps belonging to the resistance nodulation division family, 5,6 and several homologous resistance nodulation cell division-type pumps are representative in $P$. aeruginosa such that MexAB-OprM (coding gene: $\operatorname{mex} A, \operatorname{mex} B$ and $o p r M)$, MexCD-OprJ $(\operatorname{mex} C, \operatorname{mexD}$ and $o p r J)$, MexEF-OprN (mexE, mexF and oprN), MexXY(-OprA) (mexX, $m e x Y$ and oprA) and some $P$. aeruginosa strains lost oprA gene. ${ }^{7}$ MexAB-OprM contributes to antibiotic resistance to $\beta$-lactams such as cephalosporines or penicillins, macrolides, chloramphenicol, tetracycline and fluoroquinolones. ${ }^{8-10}$ MexCD-OprJ contributes to antibiotic resistance to macrolides, tetracyclines, fluoroquinolones and some $\beta$-lactams including cefepime. ${ }^{9,11,12}$ MexEF-OprN contributes to antibiotic resistance to fluoroquinolones, chloramphenicol and trimethoprim. ${ }^{13}$ MexXY-(OprA) contributes to antibiotic resistance to aminoglycosides, cefepime, ciprofloxacin and levofloxacin (LVFX). ${ }^{14}$

On the other hand, Sacha et al. stated that among the strains belonging to different clones isolated from ICUs, different levels of activity of the MexAB-OprM pump were observed. It can be assumed that this mechanism is also responsible for resistance to multiple classes of antibiotics (for example, ciprofloxacin and meropenem) in the population of $P$. aeruginosa strains colonizing hospital environments. ${ }^{15}$ Moreover, Ozer et al. reported in their intensive care units patients $86 \%$ of the isolates were determined to carry one and more resistance genes. The significant relationship between the resistance to cefepime, piperacilline/tazobactam and the mexC, that between the resistance to mezlocillin, piperacilline/tazobactam, ceftazidime, cefepime and $a m p C$, and that between the resistance to ciprofloxacin, norfloxacin and $\operatorname{oprJ}, \operatorname{oprN}$ and $n f x B$ were identified. ${ }^{16}$

We demonstrated that two or more mutations in the quinolone resistance determining the region of $\operatorname{gr} A$ and parC had a significant relationship with LVFX resistance of UTI-causing $P$. aeruginosa in the previous study. ${ }^{17}$

${ }^{1}$ Division of Urology, Department of Surgery, Kobe University Graduate School of Medicine, Kobe, Japan; ${ }^{2}$ Infection Control and Prevention, Kobe University Hospital, Kobe, Japan; ${ }^{3}$ Division of Infectious Diseases, Department of International Health, Kobe University, Graduate School of Health Science, Kobe, Japan and ${ }^{4}$ Center for Infectious Diseases, Kobe University Graduate School of Medicine, Kobe, Japan

Correspondence: Dr Assistant Professor K Shigemura, Division of Urology, Department of Surgery, Kobe University Graduate School of Medicine, 7-5-1, Kusunoki-cho, Chuo-ku, Kobe 650-0017, Japan.

E-mail: yutoshunta@hotmail.co.jp

Received 22 January 2015; revised 26 February 2015; accepted 10 March 2015; published online 8 April 2015 
Table 1 Primer sequences used in this study

\begin{tabular}{llll}
\hline Regulator or type of transporter & Gene & Forward primer(5'-3') & Reverse primer(5'-3') \\
\hline RND & $\operatorname{mexB}$ & GTGTTCGGCTCGCAGTACTC & AACCGTCGGGATTGACCTTG \\
RND & $\operatorname{mexC}$ & GTACCGGCGTCATGCAGGGTTC & TTACTGTTGCGGCGCAGGTGACT \\
RND & mexE & CCAGGACCAGCACGAACTTCTTGC & CGACAACGCCAAGGGCGAGTTCACC \\
RND & mexY & CCGCTACAACGGCTATCCCT & AGCGGGATCGACCAGCTTTC \\
Housekeeping gene & GPSL & GCAACTATCAACCGACTGGTG & GCTGTGCTCTTGCAGGTTGTG \\
\hline
\end{tabular}

Abbreviation: RND, resistance nodulation division.

In this study, we investigated the correlation between antibiotic susceptibilities and expression of the efflux pump genes in $P$. aeruginosa in UTI patients and which efflux pump gene related to antibiotic resistance, mutations of quinolone resistance genes and the patients' backgrounds, such as the presence of urinary tract underlying disease (complicated UTI) or diabetes mellitus (DM).

\section{MATERIALS AND METHODS}

\section{Bacterial isolates}

Urine cultures were obtained from patients with UTI treated at three hospitals (Kobe University Hospital, Miki City Hospital and Akashi Municipal Hospital) in Hyogo prefecture in Japan. Post-treatment isolates and other repeat isolates from the same patients were excluded from this study. A total of 105 cases of $P$. aeruginosa strains from UTI patients were investigated. The study obtained Institutional Review Board approval.

\section{Susceptibility testing}

Susceptibility testing was performed by measuring MIC for nine antibiotics: piperacillin, ceftazidime, cefozopran, aztreonam (AZT), imipenem, gentamicin, tobramycin, amikacin and LVFX, and the definition of susceptible and their ranges for concentrations tested were referred according to Clinical Laboratory Standards Institute guideline M07-A8 (Table M100-S19), using Frozen plates (Eiken Chemical Co. Ltd, Tokyo, Japan). ${ }^{18}$ The bacterial isolates were cultured as shown in the previous literature and on heart infusion agar plates at $37^{\circ} \mathrm{C}$ for $22 \mathrm{~h}$, prepared as $1 \mathrm{ml}$ of $1.0 \mathrm{McF}$ arland standard and then diluted in $9 \mathrm{ml}$ of sterile saline and inoculated into Frozen plates for $20 \mathrm{~h}$. MICs of each antibiotics were analyzed by IA 01 MIK mk II plate reader (Eiken Chemical Co. Ltd). P. aeruginosa PAO1 was used as a quality control. ${ }^{17}$

\section{RNA extraction and quantification of RNA expression using quantitative reverse-transcription PCR}

Taqman Reverse Transcription Reagents (Applied Biosystems, Carlsbad, CA, USA) were used for the synthesis of complementary DNA for all samples. Complementary DNA synthesis was performed for the amplification as follows: $48^{\circ} \mathrm{C}$ for $30 \mathrm{~min}, 95^{\circ} \mathrm{C}$ for $10 \mathrm{~min}$ and $50 \mathrm{cycle}$ of $95^{\circ} \mathrm{C}$ for $15 \mathrm{~s}$ and $62^{\circ} \mathrm{C}$ for $1 \mathrm{~min}$. One microliter of complementary DNA was used as the template in quantitative reverse-transcription PCR amplification with designed primers (Table 1) as described in previous studies. ${ }^{13,19,20} r p s L$ was used as housekeeping gene to calculate the relative level of expression of each gene. ${ }^{2}$ Quantitative reverse-transcription PCR was performed in MyiQ real-time PCR systems (BioRad, Hercules, CA, USA) according to the manufacturer's recommendation. The protocol was as follows: initial denaturation at $95^{\circ} \mathrm{C}$ for $10 \mathrm{~min}, 45$ cycles of amplification; denaturation at $95^{\circ} \mathrm{C}$ for $15 \mathrm{~s}$; annealing at $55^{\circ} \mathrm{C}$ for $5 \mathrm{~s}$, and extension at $72{ }^{\circ} \mathrm{C}$ for $10 \mathrm{~s}(\operatorname{mexB}, \operatorname{mex} Y$ and $r p s L)$ and at $95^{\circ} \mathrm{C}$ for $15 \mathrm{~min}, 40$ cycles of amplification; denaturation at $95^{\circ} \mathrm{C}$ for $20 \mathrm{~s}$; annealing at $60^{\circ} \mathrm{C}$ for $20 \mathrm{~s}$ and extension at $72{ }^{\circ} \mathrm{C}$ for $30 \mathrm{~s}$ ( $\operatorname{mexC}$ and $\operatorname{mexE}$ ). Each sample was assayed in triplicate on a $96-$ well plate.

The relative expression level of efflux pump genes was calculated by the level of relative quantification in each efflux pump gene divided by that of $r p s L$ and then the expression rate of each efflux pump gene was defined as the relative expression level of efflux pump gene in each isolate divided into greater than equal to twofold gene expression compared with that of the control (P. aeruginosa $\mathrm{PAO} 1)^{21,22}$
Table 2 Patient's characteristics of urinary tract infection

\begin{tabular}{lc}
\hline Backgrounds & $n(\%)$ \\
\hline Number & 105 \\
Age (Mean \pm s.d.) & $71.9 \pm 16.4$ \\
$\begin{array}{l}\text { Gender } \\
\text { male }\end{array}$ & \\
female & $67(63.8)$ \\
Urinary tract infection & $38(36.2)$ \\
uncomplicated & \\
complicated & $65(61.9)$ \\
Diabetes mellitus & $40(38.1)$ \\
$(-)$ & \\
$(+)$ & $86(81.9)$ \\
Previous use of LVFX & $19(18.1)$ \\
$(-)$ & \\
$(+)$ & $83(79.0)$ \\
Gene mutation (gyrA, parC) & $22(21.0)$ \\
$(-)$ & \\
$(+)$ & $66(62.9)$ \\
\hline
\end{tabular}

Abbreviation: LVFX, levofloxacin.

Association of increased expression of efflux pump genes with risk factors, each antibiotic resistance and mutations of quinolone resistance-related genes

We analyzed the association of expression of $\operatorname{mex} B, \operatorname{mex} C$, $\operatorname{mex} E$ and $\operatorname{mex} Y$ with risk factors, the MICs of each antibiotics and the presence of mutations of fluoroquinolone resistance-related genes $(\mathrm{gyrA}$ and $\mathrm{parC}){ }^{13}$

We set as the potential risk factors for $P$. aeruginosa antibiotic resistance such as gender (male), complicated UTI, DM and previous use of LVFX. Complicated UTI is defined as UTIs in which the patients had urinary tract underlying disease such as urinary tract stones, benign prostate hyperplasia or neurogenic bladder. Antibiotic resistance or the four efflux pump gene expressions tested and the mutations of fluoroquinolone resistance genes with the presence of these potential risk factors were investigated for potential correlations. We also compared the increased expression of efflux pump genes with antibiotic resistance. In addition, we confirmed the associations between increased expression of efflux pump genes and mutations of quinolone resistance-related genes.

\section{Statistical analysis}

Statistical analyses were performed by univariate and multivariate logistic regression using PASW Statistics 17.0 software packages (for Windows, SPSS Inc., Chicago, IL, USA) with $P<0.05$ considered to indicate statistical significance. Multivariable logistic regression was used to calculate odds ratios and $95 \%$ confidence intervals after controlling simultaneously for potential confounders. 


\section{RESULTS}

Patient's characteristics, sequence and efflux pump genes expressions

Patients' backgrounds are shown in Table 2. As Table 2 shows, $38.1 \%$ of all cases had complicated UTI and $18.1 \%$ of the patients had DM. The increased expressed strains were seen in $28(26.7 \%)$ in mexB, 12 $(11.4 \%)$ in $\operatorname{mexC}, 44(41.9 \%)$ in $\operatorname{mexE}$ and $40(38.1 \%)$ in $\operatorname{mex} Y$, suggesting that mexE and mexY tended to be increasingly expressed in our studies strains.

\section{Susceptibility to each antibiotics}

In 105 P. aeruginosa isolated from UTI patients, 9 (8.6\%), 9 (8.6\%), 9 (8.6\%), 28 (26.7\%), 12 (11.4\%), 21 (20.0\%), 7 (6.7\%), 10 (9.5\%) and $23(21.9 \%)$ isolates were resistant to piperacillin, ceftazidime, cefozopran, AZT, imipenem, gentamicin, tobramycin, amikacin and LVFX, respectively (Table 3 ). That is, our studied strains tended to show comparatively higher resistant ratios especially in AZT, gentamicin and LVFX (MICs and efflux pump gene expressions are shown in a Supplementary Table).

\section{Correlation of increased expression of efflux pump genes with risk} factors

We examined the association between the strains with increased expression of efflux pump genes and potential risk factors, and found

Table 3 Susceptibilities of antimicrobial agents tested

\begin{tabular}{|c|c|c|c|c|}
\hline \multirow[b]{2}{*}{ Antibiotics } & \multirow{2}{*}{$\begin{array}{c}\text { MIC range examined } \\
\left(\mu g \mathrm{ml}^{-1}\right)\end{array}$} & \multicolumn{2}{|c|}{$\begin{array}{l}\text { No. }(\%) \text { of isolates } \\
\qquad(\mathrm{n}=105)\end{array}$} & \multirow[b]{2}{*}{ Unknown } \\
\hline & & Susceptible & Resistant & \\
\hline PIPC & $<8->64$ & $95(90.4)$ & $9(8.6)$ & $1(1.0)$ \\
\hline $\mathrm{CAZ}$ & $<1->16$ & $96(91.4)$ & $9(8.6)$ & \\
\hline CZOP & $<2->16$ & $94(89.5)$ & $9(8.6)$ & $2(1.9)$ \\
\hline AZT & $<2->16$ & 77 (73.3) & $28(26.7)$ & \\
\hline IPM & $<1->8$ & 93 (88.6) & 12 (11.4) & \\
\hline GM & $<1->8$ & $84(80.0)$ & $21(20.0)$ & \\
\hline TOB & $<1->8$ & $97(92.4)$ & $7(6.7)$ & $1(1.0)$ \\
\hline AMK & $<4->32$ & $95(90.5)$ & $10(9.5)$ & \\
\hline LVFX & $<0.5->4$ & $82(78.1)$ & 23 (21.9) & \\
\hline
\end{tabular}

Abbreviations: AMK, amikacin; AZT, aztreonam; CAZ, ceftazidime; CZOP, cefozopran; GM, gentamicin; IPM, imipenem; LVFX, levofloxacin; PIPC, piperacillin; TOB, tobramycin. an association between the overexpression of mexB and mexC and complicated UTI $(P=0.001$ and $P=0.040$, respectively), and overexpression of mexC and gene mutation $(g y r A$ and $\operatorname{par} C)(P=0.033)$ by univariate analyses. In addition, we revealed a higher significant relationship between the overexpression of $\operatorname{mexB}$ and mexC, and complicated UTI $(P<0.001$ and $P=0.032$, respectively) by multivariate analyses. However, there were no significant associations between efflux pump gene expressions and gender (male), DM, previous use of LVFX and gene mutations ( $g y r A$ and parC) (Table 4). These findings suggest overexpression of $\operatorname{mex} B$ and/or mexC related to the complicated UTI.

Association of increased expression of efflux pump genes with MICs of each antibiotics

We investigated the relationship between antibiotic MICs and efflux pump genes expressions. Our statistical data demonstrated a significant association between mexC and LVFX resistance $(P=0.019)$ by univariate analyses. Multivariate analyses also showed a significant relationship between overexpression of mexC and LVFX resistance (Odds ratio $=4.48, P=0.035$ ) (Table 5), suggesting, taken together, that mexC correlated with quinolone (LVFX) resistances.

Association of increased MICs of each antibiotics and patients' backgrounds such as complicated or uncomplicated UTI, DM and previous use of LVFX

We also examined the correlation between antibiotic MICs and patients' backgrounds such as the classification of UTI, DM and previous use of LVFX. The results showed that previous use of LVFX significantly correlated with AZT and LVFX resistances (Table 6). As to AZT resistances and previous use of LVFX, it is not easy to find some reasons for this correlation; therefore, further research is necessary for this conclusion.

\section{DISCUSSION}

Among the possible mechanisms for multi-drug resistance, efflux pump systems are located in bacterial membrane transporters, and increased levels of the efflux system expression are considered to lead to antibiotic resistance. ${ }^{23}$ Of the efflux pumps, the MexAB-OprM efflux pump is expressed continuously in susceptible strains, and an increase in gene transcription is apparently sufficient for further increase of the resistance level. ${ }^{4,19,20}$ The MexR protein is the transcriptional repressor of the mexA-mexB-oprM operon. ${ }^{12}$ On the

Table 4 Correlation of overexpression of efflux pump genes with risk factors

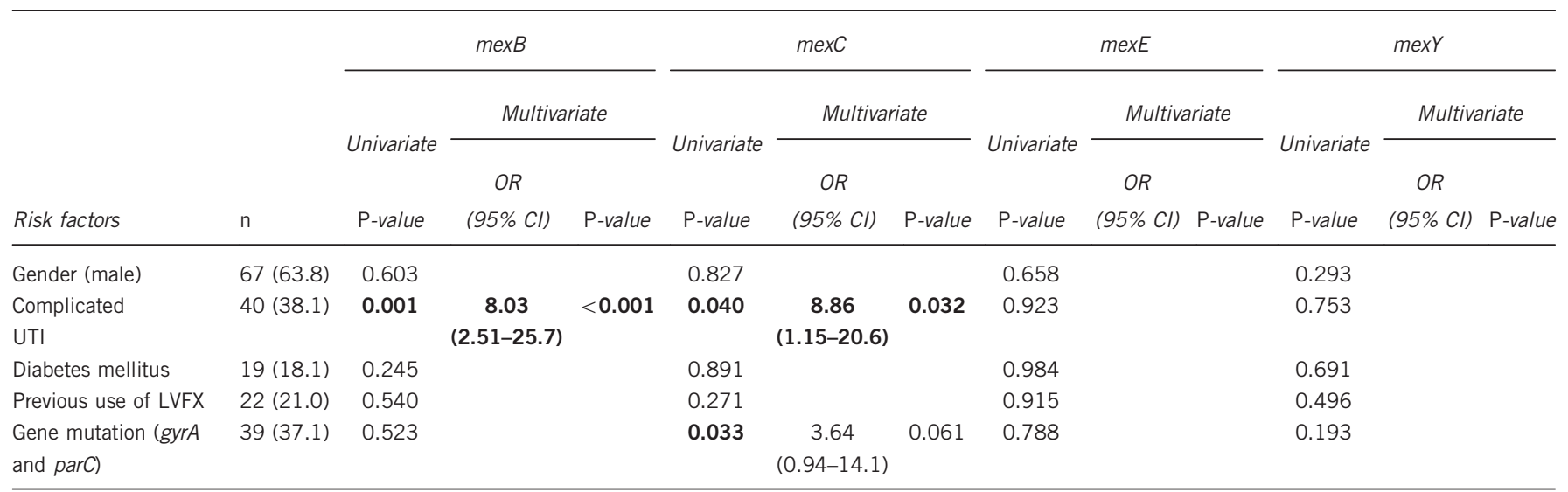

Abbreviations: $\mathrm{Cl}$, confidence interval; LVFX; levofloxacin; OR, Odds ratio; UTI, urinary tract infection.

Bold represents statistically significant values. 
Table 5 Correlation of overexpression of efflux pump genes with antibiotics susceptibilities

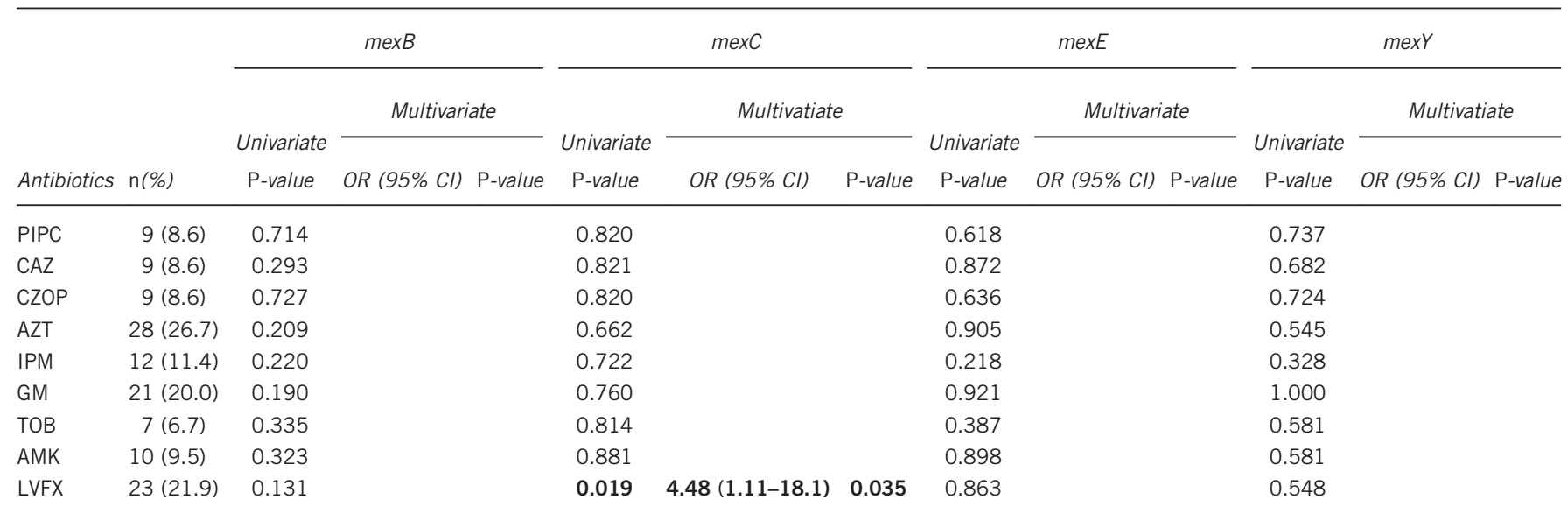

Abbreviations: AMK, amikacin; AZT, aztreonam; CAZ, ceftazidime; Cl, confidence interval; CZOP, cefozopran; GM, gentamicin; IPM, imipenem; LVFX, levofloxacin; OR, Odds ratio; PIPC, piperacillin; TOB, tobramycin.

Bold represents statistically significant values.

Table 6 Correlation of risk factors with antibiotics susceptibilities

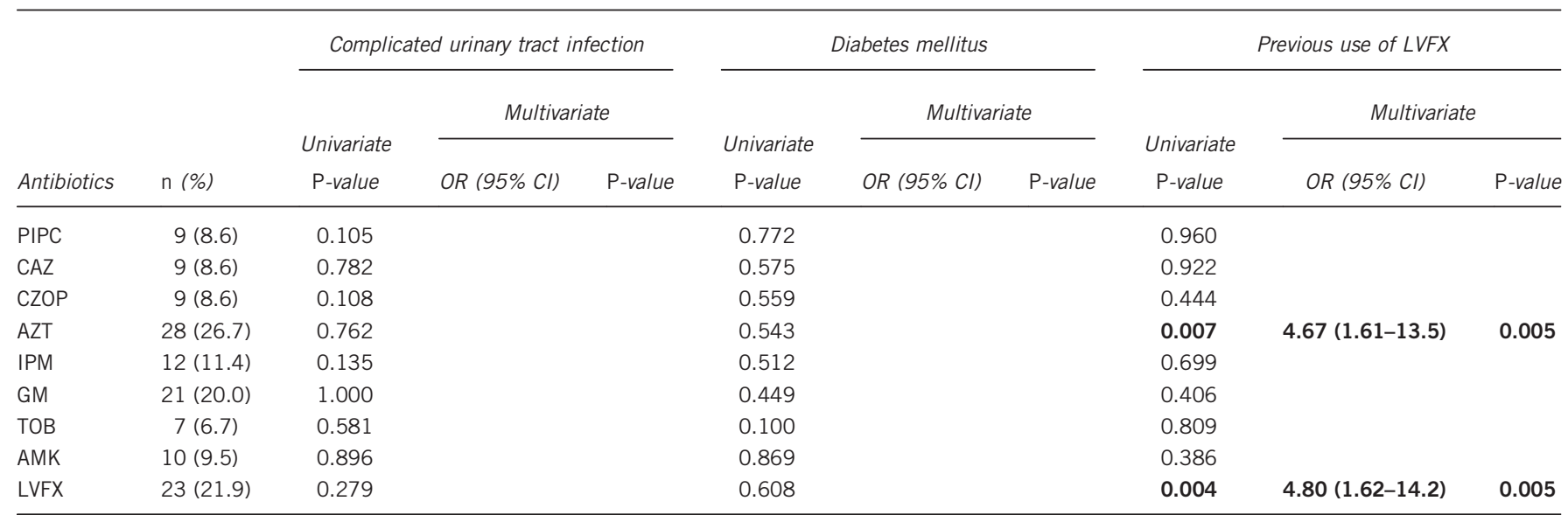

Abbreviations: AMK, amikacin; AZT, aztreonam; CAZ, ceftazidime; Cl, confidence interval; CZOP, cefozopran; GM, gentamicin; IPM, imipenem; LVFX, levofloxacin; OR, Odds ratio; PIPC, piperacillin; $\mathrm{TOB}$, tobramycin. Bold represents statistically significant values.

other hand, MexCD-OprJ and MexEF-OprN were normally silent or weakly transcribed in susceptible strains. ${ }^{24}$ We revealed that increased expression of mexC leads to antibiotic resistance to LVFX, and MexCD-OprJ contributes to resistance to fluoroquinolones, often used in UTI treatments. ${ }^{17}$

Gorgani et al. ${ }^{25}$ discovered a mutation in codon 126 of the mexR regulatory gene changing amino acid Val to Glu that correlated with fluoroquinolone resistance. Moreover, it was suggested relationship between oxacilin resistance, and expression of $\operatorname{mex} R^{26}$ and mutation in mexR gene was seen in $64 \%$ of multi-drug resistance strains, which were resistant to at least three antibiotics out of ciprofloxacin, tobramycin, ceftazidime, or imipenem. ${ }^{27}$ Masuda et al. reported that MexAB-OprM is the primary system to extrude ofloxacin in the wildtype strain and that MexXY-OprM is a compensatory system to extrude ofloxacin in the mutant lacking MexAB, ${ }^{28}$ and $\operatorname{mexE}$ (MexEFoprN) expression was reported to correlate to fluoroquinolone resistance. ${ }^{20}$ However, we were unable to detect that mexB, mexE and mexY were associated with the MIC of antibiotics including LVFX, except for mexC. As to the association of LVFX MIC and pump genes expressions, it was significant only in mexC differently from previous literatures. We speculate the reasons as follows: (1) the different materials and patients' backgrounds may produce different results related to this association. (2) The susceptibilities to fluoroquinolones such as LVFX in P. aeruginosa have been changed from epoch to epoch and from region to region in the world. (3) The use of antibiotics was also varied from region to region including dose and duration. (4) Especially, in this study, our 105 P. aeruginosa strains had $80 \%$ of susceptible strains to LVFX and this may be higher than usual population in $P$. aeruginosa causing UTI. ${ }^{29}$

There are the reports regarding the risk factors for the expressions of efflux pump genes in Escherichia coli; ${ }^{25,30}$ however, regarding $P$. aeruginosa, there were no reports of the risk factors for the expressions of efflux pump genes even though there are many reports of separate investigation of risk factors for antibiotic resistance. ${ }^{31}$ Our previous work using E. coli revealed that complicated UTI cases and female gender for LVFX resistance were significantly independent risk factors for transcriptional regulator gene marA expression. ${ }^{22}$ Our current study with $P$. aeruginosa showed that complicated UTI significantly correlated with high expressions of $\operatorname{mex} B$ and $\operatorname{mex} C$, suggesting complicated UTI may have highly expressed efflux pump genes leading to comparatively higher antibiotic MICs. Our risk factors results by multivariate analyses showed that complicated UTI 
significantly associated with increased expressions of mexE and mexC. These findings clinically indicate that complicated UTI may possibly have some effects of efflux pump inhibitors for their inhibition or killing.

Previous reports have suggested that which mutations in gyrA and parC or efflux pump expressions have a main role for fluoroquinolone resistance remains contentious. ${ }^{28,32}$ We previously showed that mutations in $g y r A$ and/or parC correlated to fluoroquinolone resistance more than efflux pump gene expressions in E. coli causing UTI without their mutual relationships. ${ }^{28}$ Our multivariate analysis data showed no significant correlation of efflux pump gene expressions with mutations in gyrA and/or parC. Taken together, these facts suggest that gyrA and parC mutations and efflux pump system were involved in fluoroquinolone resistance through other independent mechanisms in both E. coli and P. aeruginosa.

We would like to emphasize the study limitations. First, the ratio of resistant strains was comparatively fewer than in other reports and this might result in a lower level of efflux pump gene-increased expression. Second, we lacked information of history of antibiotics other than LVFX and this point might affect the significant relationship between complicated UTI and efflux pump genes expressions such as mexB and mexC. These limitations may be overcome by further studies with more ratios of resistant strains.

In conclusion, our multivariate analyses data demonstrated that overexpression of mexC efflux pump genes can lead to LVFX resistance in clinically isolated $P$. aeruginosa from UTI patients and revealed a significant relationship between the $\operatorname{mex} B$ or $\operatorname{mex} C$ efflux pump genes and complicated UTI. These results contribute to our knowledge of the efflux pump system.

\section{ACKNOWLEDGEMENTS}

We thank Dr Masanori Kameoka for technical support. This study was supported by a grant-in-aid from the Program of Founding Research Centers for Emerging and Reemerging Infectious Diseases, the Ministry of Education, Culture, Sports, Science and Technology, Japan.

1 Hsueh, P. R. et al. Consensus review of the epidemiology and appropriate antimicrobia therapy of complicated urinary tract infections in Asia-Pacific region. J. Infect. 63 114-123 (2011).

2 Lu, P. L. et al. Epidemiology and antimicrobial susceptibility profiles of Gram-negative bacteria causing urinary tract infections in the Asia-Pacific region: 2009-2010 results from the Study for Monitoring Antimicrobial Resistance Trends (SMART). J. Antimicrob. Agents 40, S37-S43 (2012).

3 Xiao, M. et al. Antimicrobial susceptibility of Pseudomonas aeruginosa in China: a review of two multicentre surveillance programmes, and application of revised CLS susceptibility breakpoints. Int. J. Antimicrob. Agents 40, 445-449 (2012).

4 Lister, P. D., Wolter, D. J. \& Hanson, N. D. Antibacterial-resistant Pseudomonas aeruginosa: clinical impact and complex regulation of chromosomally encoded resistance mechanisms. Clin. Microbiol. Rev. 22, 582-610 (2009).

5 Piddock, L. J. Multidrug-resistance efflux pumps-not just for resistance. Nat. Rev. Microbiol. 4, 629-636 (2006)

6 Poole, K. Multidrug efflux pumps and antimicrobial resistance in Pseudomonas aeruginosa and related organisms. J. Mol. Microbiol. Biotechnol. 3, 255-264 (2001)

7 Morita, Y., Tomida, J. \& Kawamura, Y. Primary mechanisms mediating aminoglycoside resistance in the multidrug-resistant Pseudomonas aeruginosa clinical isolate PA7. Microbiology 158, 1071-1083 (2012).
8 Li, X. Z., Nikaido, H. \& Poole, K. Role of MexA-MexB-OprM in antibiotic efflux in Pseudomonas aeruginosa. Antimicrob. Agents Chemother. 39, 1948 (1995).

9 Li, X Z Barré, N. \& Poole, K Influence of the MexA-MexB-oprM multidrug efflux system on expression of the MexC-MexD-oprJ and MexE-MexF-oprN multidrug efflux systems in Pseudomonas aeruginosa. J. Antimicrob. Chemother. 46 885-893 (2000).

10 Poole, K., Krebes, K., McNally, C. \& Neshat, S. Multiple antibiotic resistance in Pseudomonas aeruginosa: evidence for involvement of an efflux operon. J. Bacteriol. 175, 7363-7372 (1993).

11 Morita, $Y$. et al. Induction of mexCD-oprJ operon for a multidrug efflux pump by disinfectants in wild-type Pseudomonas aeruginosa PAO1. J. Antimicrob. Chemother. 51, 991-994 (2013).

12 Poole, K. et al. Overexpression of the mexC-mexD-oprJ efflux operon in nfxB-type multidrug-resistant strains of Pseudomonas aeruginosa. Mol. Microbiol. 21 713-724 (1996)

13 Köhler, T. et al. Characterization of MexE-MexF-OprN, a positively regulated multidrug efflux system of Pseudomonas aeruginosa. Mol. Microbiol. 23, 345-354 (1997).

14 Muller, C., Plésiat, P. \& Jeannot, K. A two-component regulatory system interconnects resistance to polymyxins, aminoglycosides, fluoroquinolones, and $\beta$-lactams in Pseudomonas aeruginosa. Antimicrob. Agents Chemother. 55, 1211-1221 (2011).

15 Sacha, P. et al. Expression of MexAB-OprM efflux pump system and susceptibility to antibiotics of different Pseudomonas aeruginosa clones isolated from patients hospitalized in two intensive care units at University Hospital in Bialystok (northeastern Poland) between January 2002 and December 2009. APMIS 122, 931-940 (2014).

16 Aeschlimann, J. R. The role of multidrug efflux pumps in the antibiotic resistance of Pseudomonas aeruginosa and other gram-negative bacteria. Pharmacotherapy 23 916-924 (2003).

17 Matsumoto, M. et al. Mutations in the gyrA and parC genes and in vitro activities of fluoroquinolones in 114 clinical isolates of Pseudomonas aeruginosa derived from urinary tract infections and their rapid detection by denaturing high-performance liquid chromatography. Int. J. Antimicrob. Agents 40, 440-444 (2012).

$18 \mathrm{CLSI}$-Clinical Laboratory Standards Institute. Performance standards for antimicrobial susceptibility testing: M100-S19. Clinical Laboratory Standards Institute. Wayne, PA (2009).

19 Fernández, L. \& Hancock, R. E. Adaptive and mutational resistance: role of porins and efflux pumps in drug resistance. Clin. Microbiol. Rev. 25, 661-681 (2012).

20 Poole, K. Pseudomonas aeruginosa: resistance to the max. Front. Microbiol. 2, 65 (2011).

21 Tomás, M. et al. Efflux pumps, OprD porin, AmpC beta-lactamase, and multiresistance in Pseudomonas aeruginosa isolates from cystic fibrosis patients. Antimicrob. Agents Chemother. 54, 2219-2224 (2010).

22 Yasufuku, T. et al. Correlation of overexpression of efflux pump genes with antibiotic resistance in Escherichia coli strains clinically isolated from urinary tract infection patients. J. Clin. Microbiol. 49, 189-194 (2011).

23 Ozer, B., Duran, N., Onlen, Y. \& Savas, L. Efflux pump genes and antimicrobial resistance of Pseudomonas aeruginosa strains isolated from lower respiratory tract infections acquired in an intensive care unit. J. Antibiot. (Tokyo) 65, 9-13 (2012).

$24 \mathrm{Han}, \mathrm{J} . \mathrm{H}$. et al. Risk factors for efflux pump overexpression in fluoroquinolone-resistant Escherichia coli. J. Infect. Dis. 206, 1597-1603 (2012).

25 Gorgani, N., Ahlbrand, S., Patterson, A. \& Pourmand, N. (2009) Detection of point mutations associated with antibiotic resistance in Pseudomonas aeruginosa. Int. J. Antimicrob. Agents 34, 414-4182009.

26 Sadeghifard, N. et al. Relationship between the presence of the nalC mutation and multidrug resistance in Pseudomonas aeruginosa. Int. J. Microbiol. 2012 575193 (2012).

27 Kiser, T. H., Obritsch, M. D., Jung, R., MacLaren, R. \& Fish, D. N. Efflux pump contribution to multidrug resistance in clinical isolates of Pseudomonas aeruginosa. Pharmacotherapy 30, 632 (2010)

28 Shigemura, K. et al. Does mutation in gyrA and/or parC or efflux pump expression play the main role in fluoroquinolone resistance in Escherichia coli urinary tract infections?: A statistical analysis study. Int. J. Antimicrob. Agents 40, 516-520 (2012).

29 Masuda, N. et al. Contribution of the MexX-MexY-oprM efflux system to intrinsic resistance in Pseudomonas aeruginosa. Antimicrob. Agents Chemother. 44 2242-2246 (2000).

30 Morgan-Linnell, S. K., Becnel Boyd, L., Steffen, D. \& Zechiedrich, L. Mechanisms accounting for fluoroquinolone resistance in Escherichia coli clinical isolates. Antimicrob. Agents Chemother. 53, 235-241 (2009).

$31 \mathrm{Tam}, \mathrm{V}$. H. et al. Prevalence, mechanisms, and risk factors of carbapenem resistance in bloodstream isolates of Pseudomonas aeruginosa. Diagn. Microbiol. Infect. Dis. 58, 309-314 (2007).

32 Pasca, M. R. et al. Evaluation of fluoroquinolone resistance mechanisms in Pseudo monas aeruginosa multidrug resistance clinical isolates. Microb. Drug Resist. 18 23-32 (2012).

Supplementary Information accompanies the paper on The Journal of Antibiotics website (http://www.nature.com/ja) 